\title{
Chloromethyl acryl reagents for simple and site-selective cysteine and disulfide modification
}

Lujuan Xu, Seah Ling Kuan*, and Tanja Weil*

\begin{abstract}
The generation of protein biotherapeutics with improved features compared to the synthetic drugs has received emerging interest. The conjugation of various synthetic functionalities to proteins provides access to new classes of protein conjugates, where the advantages from both the synthetic world and Nature can be combined in a synergistic fashion. Here, we reported that 2-chloromethyl acryl scaffold can serve as a simple yet versatile platform for synthesizing acrylamide or acrylate derivatives by coupling with different end-group functionalities (amino group or hydroxyl group) via a one-pot reaction. The chemical properties of the amide or ester linkage influence their inherent reactivity as bioconjugation reagents, which in turn allows synthetic customization of their features to achieve selective protein modification at cysteine or disulfide sites on demand. 2-Chloromethyl acrylamide reagents with amide linkage favors selective modification at cysteine site with high efficiency and the resultant bioconjugates exhibit superior stability compared to commonly employed maleimide-thiol conjugates. In contrast, 2-chloromethyl acrylate reagents bearing ester linkage can undergo two successive Michael reaction, allowing the selective modification of disulfides with high labelling efficiency and conjugate stability. These reagents could outperform widely applied maleimide reagents in terms of stability of the resultant bioconjugates without compromising on the ease of reagent preparation, reactivity and reaction speed.
\end{abstract}




\section{Introduction}

Proteins are an emerging class of biotherapeutics with high target affinity and specificity, as exemplified by monoclonal antibodies that are applied for treating several severe diseases such as multiple sclerosis or cancer. ${ }^{1-3}$ Site-selective modification of proteins enables the incorporation of desired synthetic functionalities into proteins at distinct sites, which greatly enriches their structural and functional diversity. ${ }^{4-7}$ In this manner, the vital link between the synthetic world and Nature can be bridged to combine the advantages from both worlds for the construction of protein bioconjugates with high activity, structural perfection and novel functional characteristics. $^{8-10}$

Chemical approaches for protein modification allow the straightforward attachment of one or more functionalities at natural amino acid residues on the protein surface, thereby eliminating the need for tedious genetic engineering and also significantly mitigating the risk of negative effects on proteins folding and loss of function. ${ }^{11}$ The amino acids with distinct reactivity profiles and low abundance on the protein surface are considered as primary choices for residue-specific modifications. ${ }^{11}$ Among these, unpaired cysteines are considered as one of the most sought after targets owing to the high nucleophilicity and versatile chemistry landscapes of thiol groups. ${ }^{12-15}$ In addition, disulfide bonds, the oxidized form of cysteine residues, have also emerged as attractive modification sites to incorporate tailored functionalities, as a lot of therapeutic relevant proteins or peptides, e.g. antibody or its antigen-binding fragments, contain at least one solvent accessible disulfide bond. ${ }^{16-17}$

Maleimide reagents have emerged as the most widely applied cysteine bioconjugation tool due to their fast and efficient reactions with thiols. ${ }^{12}$ Besides that, a variety of structurally diverse reagents including vinylpyrimidine reagents ${ }^{18}$, carbonylacrylic reagents ${ }^{19}$ and vinylphosphonites ${ }^{20}$, among many others, have been reported for the selective cysteine modification ${ }^{21-22}$. However, the strategies for disulfide modification are much less explored and the current toolset is limited to five to six conjugation methods available in the literature, including bis- and allyl sulfones ${ }^{23-25}$, dibromomaleimide derivatives ${ }^{26-27}$, divinylpyridines ${ }^{28}$ and oxetane reagents ${ }^{29}$. The stark contrast in the availability of disulfide functionalization strategies, compared to free cysteine modification, stems from the stringent requirement to simultaneously react with two cysteine residues in a concerted fashion using a single reagent in order to preserve structure and bioactivity. Even though significant progress has been made in the development of new methodologies for protein modification at cysteine or disulfide sites, many of these strategies are still limited by complicated multi-step synthesis, poor solubility and biocompatibility of the conjugation reagents, or the limited stability of the resultant 
bioconjugates. In addition, general accessibility and scalability of the bioconjugation reagents for industrial applications are still severely restricted. Moreover, there is an increasing need to attach functionalities at two different sites on the surface of proteins to enhance multiple features; e.g. stability and traceability. ${ }^{9}$ However, the reagents developed to date mainly target a single amino acid residue, for example a cysteine residue or a disulfide bond. Besides the (bromo)maleimides ${ }^{26}$, 3-bromo-5-methylene pyrrolones ${ }^{30}$ and diethynyl phosphinates ${ }^{31}$, there are only a few bioconjugation reagents that can provide a broad spectrum scaffold to address both cysteine and disulfide sites in a user-defined fashion with high labeling efficiency. Such a strategy is more advantageous compared to reinvent a different scaffold for every single purpose. In this way, the synthetic path is more straightforward if both the cysteine and disulfide modification need to be achieved for applications. Therefore, the development of such bioconjugation reagents would be highly advantageous to enrich the existing toolbox and also enable non-experts to conduct such protein labeling reactions.

In this work, we report 2-chloromethyl acryl reagents that serve as an easily accessible yet versatile scaffold for synthesizing acrylamide or acrylate reagents in a one-pot reaction by coupling with different endgroup functionalities (amino group or hydroxyl group) (Fig. 1). Specifically, the inherent reactivity of the bioconjugation reagents can be customized by exploiting the different electron-withdrawing properties of an amide or ester linkage to address a broad spectrum of peptides and proteins that contain either a free cysteine or accessible disulfide bonds. We showed herein that 2-chloromethyl acrylamide compounds containing an amide bond in the scaffold can react with proteins containing a free thiol group via a single Michael reaction with near quantitative conversions. In contrast to maleimide conjugation, the 2-chloromethyl acrylamide reagents and the resultant bioconjugates showed superior stability. By replacing the amide with an ester linkage yielding the respective 2-chloromethyl acrylate reagents, site-selective disulfide modification can be achieved as exemplified by successful modification of three disulfide-containing substrates. The bioconjugation reagents reported herein are characterized by facile linker synthesis, high water solubility and labelling efficiency as well as superior stability of the resultant protein bioconjugates. 


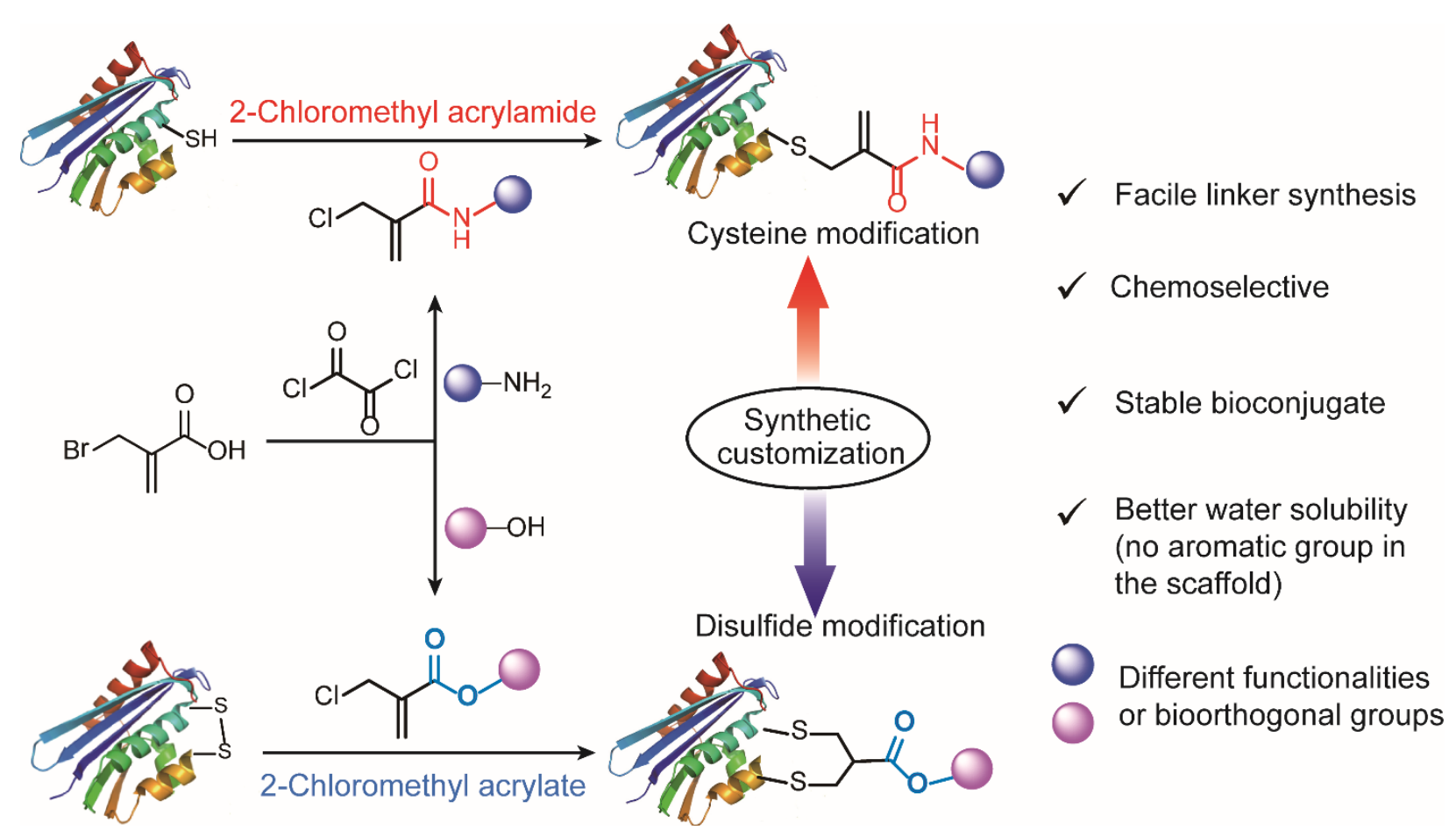

Fig. 1 General scheme for 2-chloromethyl acrylamide and acrylate compounds for site-selective protein modification at cysteine or disulfide sites via synthetic customization.

\section{Results and discussion}

\section{Synthesis of 2-chloromethyl acrylamide and acrylate compounds}

Inspired by the inherent features of the electron-deficient systems serving as good Michael acceptors for the reactions with nucleophiles on the protein surface ${ }^{12}$, we reasoned that 2-halomethyl acryl derivatives (acrylamide or acrylate) will serve as an appropriate option for reactions with cysteine residues to accomplish site-selective protein modification. This is due to the fact that 2-halomethyl acryl derivatives contain a carbonyl group as electron-withdrawing moiety and a halogen atom as good leaving group (Fig. S1). After the release of the halogen atom, the acrylamide or acrylate group can still serve as an additional Michael acceptor for subsequent reactions and consequently, accomplish bis-alkylation for disulfide modification. Moreover, considering the different electron withdrawing properties of the ester and amide bond, we further speculated that the reactivity profile of the second Michael reaction will be influenced by their different behaviors as electrophiles.

We initiated our study by using the commercially available compound, 2-(bromomethyl)acrylic acid, as starting material to synthesize both 2-halomethyl acrylamide and acrylate bioconjugation reagents (Scheme 1 and S1). First, 2-(bromomethyl)acrylic acid reacted with oxalyl chloride to convert the carboxylic acid group to acid chloride in situ. Thereafter, different end-group nucleophiles, amino or alcohol groups (usually 1.5 to 
2 equiv.) were added under basic conditions for further reactions. The respective 2-chloromethyl acrylamide and acrylate were subsequently purified and isolated in moderate yields (Scheme 1). Mass spectrometry (MS) data demonstrated that the bromine atom is completely replaced by the chlorine atom affording the 2chloromethyl acryl compounds (Fig. S38-S43). A toolbox containing different functionalities, e.g. dye or bioorthogonal groups, was obtained as demonstrated in Scheme 1 underlining the broad applicability of this method. Compared to other disulfide- and cysteine-modification reagents, which require multiple-step synthesis (e.g., allyl sulfones requires five-step synthesis ${ }^{23}$ ), the 2-chloromethyl acryl derivatives are readily available through a straightforward one-pot synthesis from commercially available 2-(bromomethyl)acrylic acid precursors. The simplicity of the synthesis provides fast and efficient access to a broad spectrum of functionalities that are of great interest in protein bioconjugates.

In addition, compared to the reported cysteine and disulfide modification reagents, e.g. carbonylacrylic reagent ${ }^{19}$ or ally sulfone reagents ${ }^{23}$ that contain a hydrophobic phenyl group, the 2-chloromethyl acryl derivatives do not contain any aromatic group in the scaffold, which provides lower partition coefficient $(n$ octanol to water, log $\mathrm{P}_{\mathrm{o} / \mathrm{w}}$ ) (Fig. S2-S3) indicating improved water-solubility. Furthermore, the stability of the bioconjugation reagents in different aqueous environments represents an important consideration for their subsequent usage. The stability of the 2-chloromethyl acrylamide and acrylate compounds was evaluated by incubating compound $\mathbf{3}$ and compound $\mathbf{4}$ at three different $\mathrm{pH}(\mathrm{pH} \mathrm{6,7}$ and 8), and the HPLC data indicated that they remained stable over a time course of 36 hours without any degradation (Fig. S4-S9). In contrast, maleimides reagents, which are the most commonly used bioconjugation reagents for cysteine functionalization, easily hydrolyze to nonreactive maleic amides, especially at basic $\mathrm{pH}\left(\mathrm{t}_{1 / 2}<\mathrm{two}\right.$ hours) (Fig. S10-S12). 


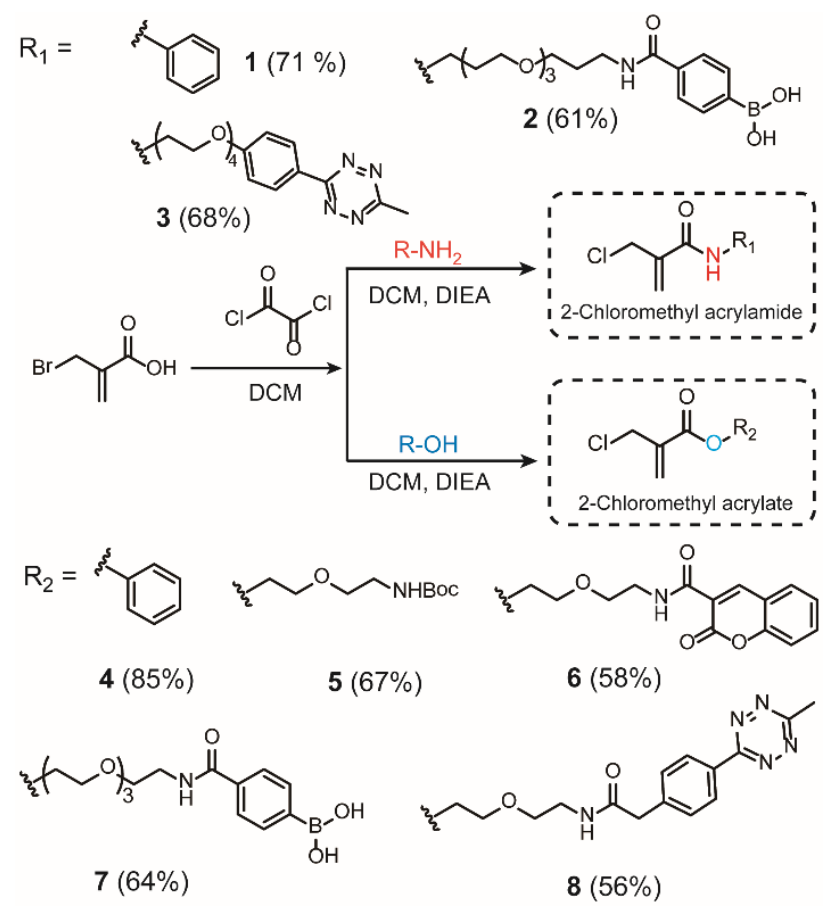

Scheme 1 Synthesis route for 2-chloromethyl acrylamide and acrylate derivatives containing different functionalities.

\section{Chemoselectivity of 2-chloromethyl acrylamide and acrylate towards thiol groups}

The reactivity and selectivity of 2-chloromethyl acrylamides and acrylates towards two model amino acids: Boc-Cys-OMe and Boc-Lys-OH (Fig. 2) were evaluated first. For 2-chloromethyl acrylamide, compound 1 was incubated with both Boc-Cys-OMe and Boc-Lys-OH in acetonitrile (ACN) / phosphate buffer (PB, pH 7) for four hours (Fig. 2a). Liquid chromatography (LC) data indicated quantitative conversion to the cysteinemodified compound $\mathbf{1 0}$ while lysine-modified compound $\mathbf{9}$ was not observed, which clearly demonstrated its excellent chemoselectivity towards cysteine over lysine residues (Fig. 2c). Compound 10 did not undergo any further reaction even in the presence of excess of Boc-Cys-OMe (8 equiv.) (Fig. 2d). No further reactions were observed even when the reaction temperature was increased to $37^{\circ} \mathrm{C}$ or after stirring for days.

For 2-chloromethyl acrylate, compound $\mathbf{4}$ was incubated with both Boc-Cys-OMe and Boc-Lys-OH under the same conditions used for compound 1 (Fig. 2b). The LC trace also revealed the excellent chemoselectivity towards thiol groups as lysine-modified compound $\mathbf{1 1}$ was also not observed in the mixture (Fig. 2e). However, in contrast to the reaction with acrylamides, with the increasing amounts of Boc-Cys-OMe the peak for compound 12 decreased while the signal for compound 13 increased (Fig. 2f). After adding eight equivalent of Boc-Cys-OMe, compound 4 was fully converted to compound 13 with negligible side product formation (Fig 2f). 
These model reactions clearly indicated a pronounced difference in the reactivity of the 2-chloromethyl acrylamide versus the acrylate reagents, presumably originating from the amide or ester linkage. It was reported that catalysts and high temperature are requivalentsuired for thiol addition with $\alpha$, $\beta$-unsaturated amides as Michael acceptors. ${ }^{32-33}$ Therefore, we postulated that the second Michael reaction of the 2chloromethyl acrylamide did not proceed due to the relatively weak electron-withdrawing property of the amide bond, which rendered the $\alpha, \beta$-unsaturated amide a poor Michael acceptor. ${ }^{30}$ Taken together, these results demonstrated that 2-chloromethyl acrylamides allow straightforward modification of free cysteines with high efficiency and excellent chemoselectivity. In contrast, 2-chloromethyl acrylates can undergo two Michael reactions in a successive manner, thereby making them suitable candidates to achieve protein modification at the disulfide sites.
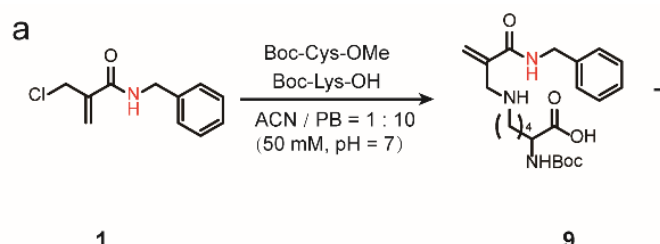

9

b

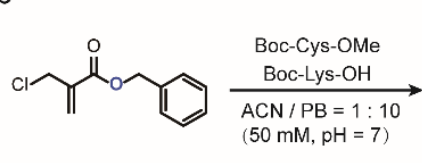
4

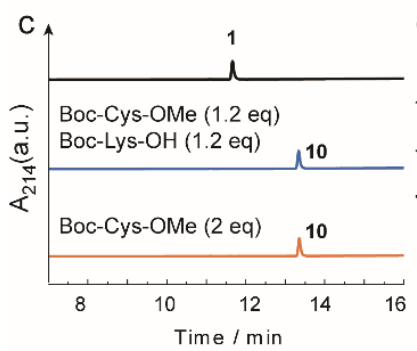

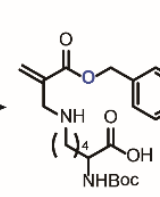

11

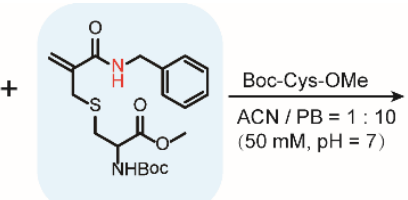

10

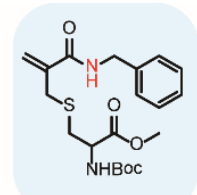

10

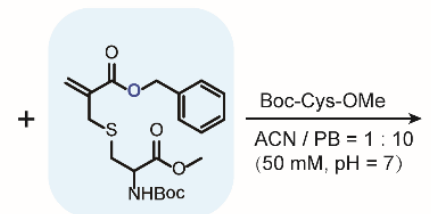

12

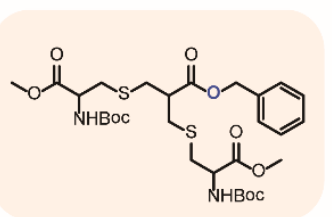

13
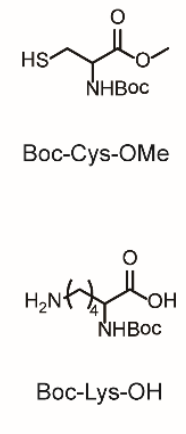

Fig. 2 (a) Reaction scheme between 2-chloromethyl acrylamide and Boc-Cys-OMe (and Boc-Lys-OH). (b) Reaction scheme between 2-chloromethyl acrylate and Boc-Cys-OMe (and Boc-Lys-OH). (c) LC trace of the reaction between 2-chloromethyl acrylamide and Boc-Cys-OMe (and Boc-Ly-OH). (d) LC trace of 2chloromethyl acrylamide with Boc-Cys-OMe (from 4 equiv. to 8 equiv.). (e) LC trace of the reaction between 2-chloromethyl acrylate and Boc-Cys-OMe (and Boc-Lys-OH) (c) LC trace of 2-chloromethyl acrylate and Boc-Cys-OMe (from 2 equiv. to 8 equiv.)

\section{2-Chloromethyl acrylamide reagents for cysteine modification}

Next, the reaction kinetic was first studied using a model reaction between compound $\mathbf{3}$ and Boc-Cys- 
OMe (Fig. 3a). Compound 3 (1 mM) and Boc-Cys-OMe (1 mM) were incubated in ACN / PB (pH 7) mixture (volume ratio: $1: 10$ ) using Fmoc-Phe-OH (Scheme S3) as internal standard. At different time intervals, the reaction was monitored by high-performance liquid chromatography (HPLC) (Fig. S16), and quantification of compounds 3 and 14 over time was plotted with reference to the internal standard (Fig. 3b). HPLC analysis indicated that $80 \%$ of compound $\mathbf{3}$ was converted to the cysteine-modified compound $\mathbf{1 4}$ in less than two hours and near quantitative conversion was achieved in less than six hours (Fig. S16). The second-order rate constant was determined to $1.17 \mathrm{M}^{-1} \mathrm{~s}^{-1}$ with the concentration of compound 3 at $1 \mathrm{mM}$ (Fig. 3c). Although this reaction is slower than the maleimide conjugation $\left(10-1000 \mathrm{M}^{-1} \mathrm{~s}^{-1}\right)^{34}$, it is still comparable to or even faster than the recently reported bioconjugation reagents, e.g. ethynylphosphonamidates $\left(0.62 \mathrm{M}^{-1} \mathrm{~s}^{-1}\right)^{35}$, and some other conventional bioconjugation methods such as oxime ligation $\left(0.001 \mathrm{M}^{-1} \mathrm{~s}^{-1}\right)^{36-37}$, Pictet-Spengler ligation $\left(0.015 \mathrm{M}^{-1} \mathrm{~s}^{-1}\right)^{34}$ and strain-promoted azide-alkyne reaction $\left(0.9 \mathrm{M}^{-1} \mathrm{~s}^{-1}\right)^{38}$.

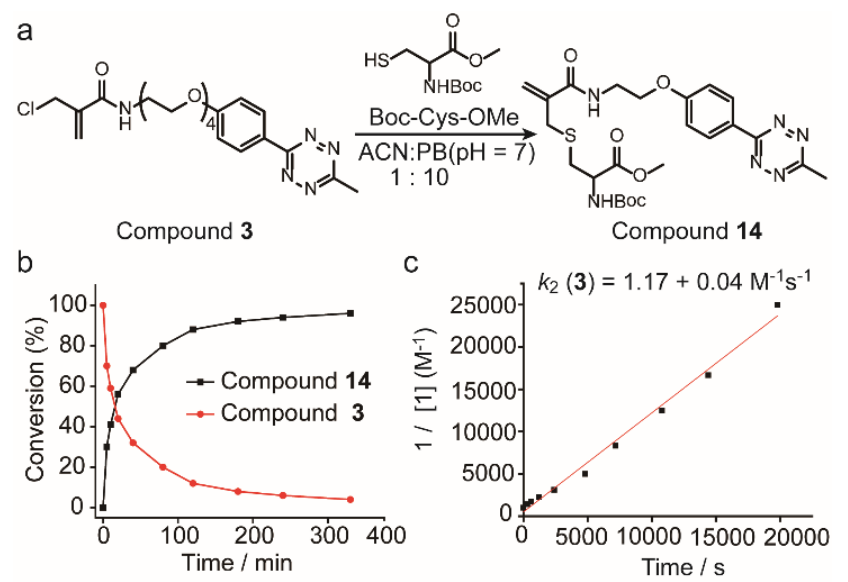

Fig. 3 (a) Reaction scheme of compound 3 reacting with Boc-Cys-OMe to form compound 14. (b) Percentage of compound $\mathbf{3}$ and $\mathbf{1 4}$ as determined by the integration of the HPLC peak in comparison to the internal standard at different time points. (c) Experimental determination of the second-order rate constant of the model reaction between compound $3(1 \mathrm{mM})$ and Boc-Cys-OMe $(1 \mathrm{mM})$. Details about the calculation and kinetics data were demonstrated in Section 4 in SI.

Thereafter, 2-chloromethyl acrylamide derivatives were applied for cysteine modification on peptide substrates using compound 3 (Fig. 4a). First, the known WSC02 peptide (sequence:IVRWSKKVCQVS), an endogenous peptide inhibitor of the chemokine CXCR4 receptor, which is highly relevant for anti-infectivity in viral infection and anti-migratory effect in cancer ${ }^{39}$, was selected as bioactive substrate (Fig. 4b). In ACN / PB mixture (1: 10), one equivalent WSC02 peptide was incubated with 1.1 equivalents of compound 3 for four hours. HPLC analysis of the crude reaction mixture indicated that more than $95 \%$ conversion to the desired modified product (WSC02-PEG4-Tz) was achieved (Fig. 4c). As control, the thiol reactive reagent 4,4'- 
dithiodipyridine (4-DPS), which is often used for free thiol quantification on proteins via a thiol-disulfide exchange reaction (the reaction mechanism is shown in Scheme S5) ${ }^{40}$, was used to mask the cysteine residue. In this case, no further reaction was observed in the HPLC chromatogram in the presence of compound 3 under the same reaction conditions (Fig. 4c). Taken together, these data clearly indicated that the 2chloromethyl acrylamide compounds exhibit excellent chemoselectivity in combination with excellent modification efficiency. In addition, it was reported that the thiol-maleimide conjugates are prone to decomposition via hydrolysis and/or retro-Michael reaction. ${ }^{41}$ Therefore, the stability of the obtained WSC02PEG4-Tz conjugate was evaluated, and it was shown that the conjugate remained stable for up to 36 hours in aqueous solutions, and no loss of the tetrazine groups was observed (Fig. S20). In addition to WSC02, five other peptides, including RGDC, CEIE, PC-8, Tet and EK-1 peptides (sequences and MS of the modified peptides were shown in Fig. 4e and Fig. S21-S25), have also been successfully modified with compound 3. The broad range of substrates used here clearly demonstrate the general applicability of 2-chloromethyl acrylamide compounds for site-selective cysteine modification.

After demonstrating the successful modification of the model peptides, we proceeded to functionalize the more complex protein substrates. Therefore, the protein ubiquitin that plays an important role in protein degradation by the proteasome was selected, which contains a cysteine mutation at its K63 position (Fig. 5a). After incubation of one equivalent ubiquitin with ten equivalents of two different 2-chloromethyl acrylamide derivatives in $\mathrm{PB}(\mathrm{pH}=7)$ for four hours, the desired bioconjugates were obtained. The successful modification was confirmed with the expected $\mathrm{m} / \mathrm{z}$ in the MS shown in Fig. 5b. Similarly, if 4-DPS was used to mask the accessible cysteine residue on the protein surface, no reaction was observed even in the presence of ten equivalents of the 2-chloromethyl acrylamides (Fig. S26). Besides ubiquitin, a single-chain $\mathrm{V}_{\mathrm{H}} \mathrm{H}$ antibody domain with specific binding activity against the green fluorescent protein (anti-GFP nanobody) has also been successfully modified with 2-chloromethyl acrylamide derivatives via using the same reaction conditions described above (Fig. 5c). The MALDI-Tof-MS characterization clearly indicated the successful modification with the expected $\mathrm{m} / \mathrm{z}$ shown in Fig. $5 \mathrm{~d}$. 
a
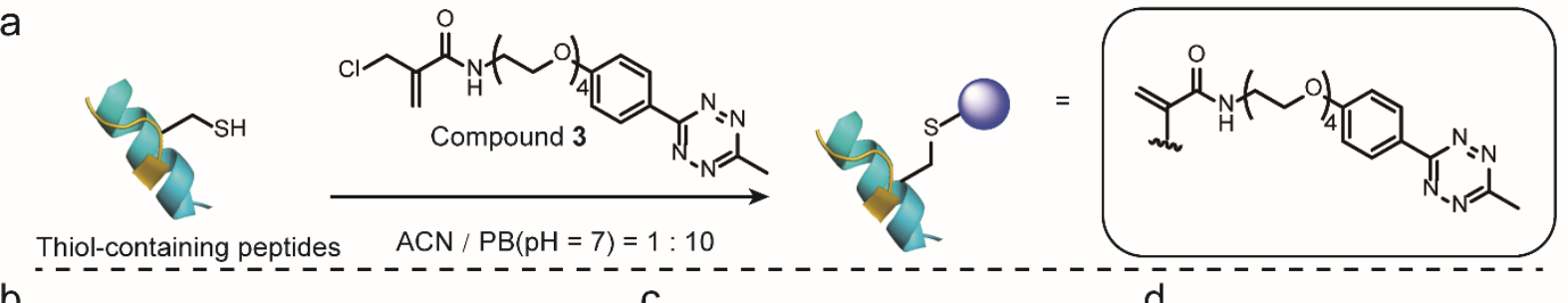

$\mathrm{b}$
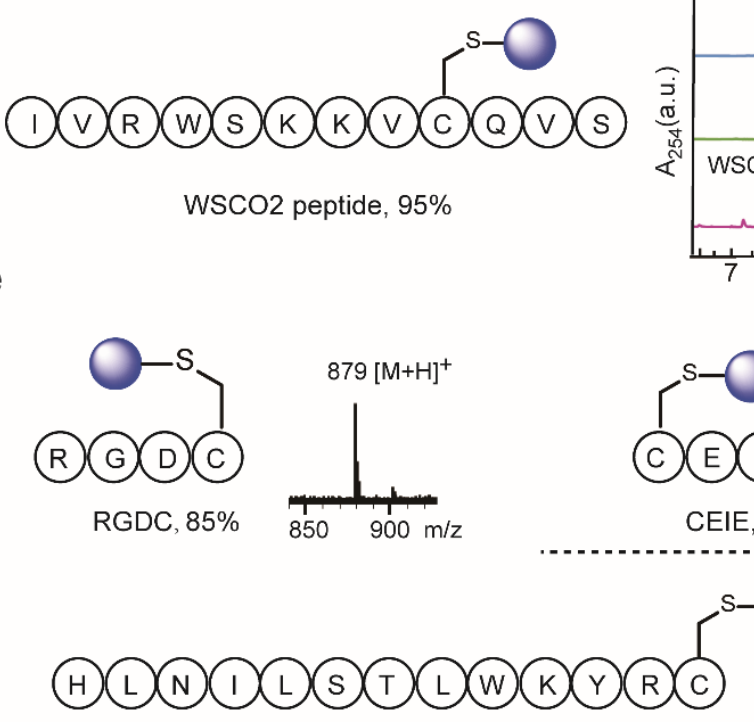

Tet peptide, $83 \%$

\footnotetext{
e

\section{WSCO2 peptide, $95 \%$}

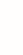

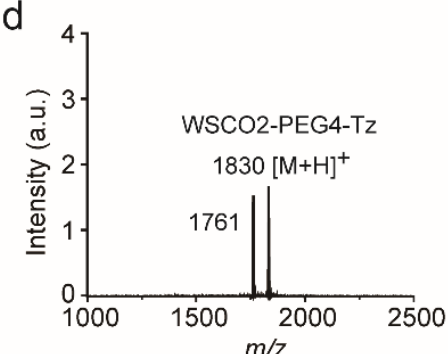

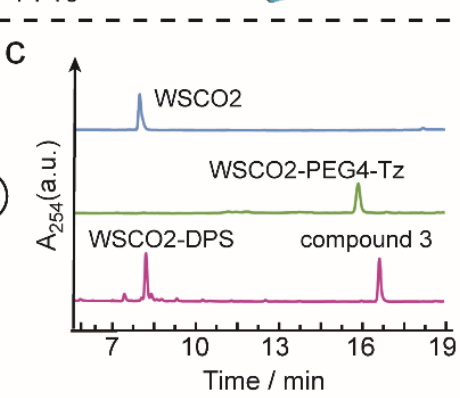
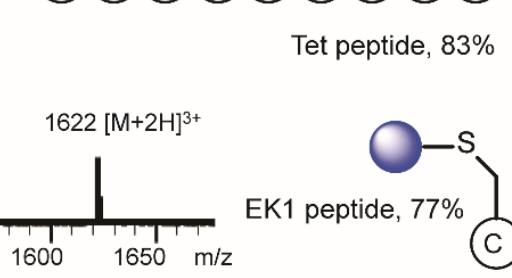

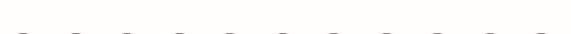

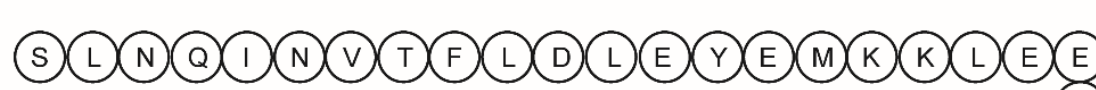

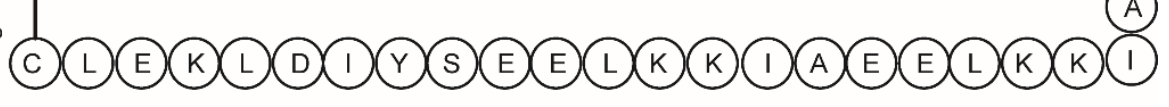

Fig. 4. (a) General scheme for site-selective peptide modification with compound 3 in ACN / PB ( $\mathrm{pH} 7$ ) mixture. (b) WSCO2 peptide was selected as model substrate for modification at cysteine site with compound 3. (c) HPLC trace of WSCO2 peptide, crude reaction mixture between WSCO2 and compound 3, WSCO2-DPS and compound 3 (from top to down) which demonstrated the efficient modification efficiency and good chemoselectivity. (d) MALDI-Tof-MS of modified WSCO2 peptide showing a signal at 1830 which is attributed to the WSCO2-PEG4-Tz (calculated: $1830[\mathrm{M}+\mathrm{H}]^{+}$, found: $1830[\mathrm{M}+\mathrm{H}]^{+}$). The signal at 1761 corresponded to the fragmentation product with the double bond breaking at tetrazine moiety, the chemical structure is shown in SI. (e) Site-selective modification of different thiol-containing peptides including RGDC (calculated: 879 $[\mathrm{M}+\mathrm{H}]^{+}$, found: $\left.879[\mathrm{M}+\mathrm{H}]^{+}\right)$, CEIE (calculated: $922[\mathrm{M}+\mathrm{H}]^{+}$, found: $\left.922[\mathrm{M}+\mathrm{H}]^{+}\right), \mathrm{PC}-8\left(\right.$ calculated: $1417[\mathrm{M}+\mathrm{H}]^{+}$, found: $\left.1417[\mathrm{M}+\mathrm{H}]^{+}\right)$, Tet peptide (calculated: $1125[\mathrm{M}+2 \mathrm{H}]^{2+}$, found: $1125[\mathrm{M}+2 \mathrm{H}]^{2+}$ ) and EK1 peptide (calculated: $1622[\mathrm{M}+3 \mathrm{H}]^{3+}$, found: $1622[\mathrm{M}+3 \mathrm{H}]^{3+}$ ) with a tetrazine group. 
a

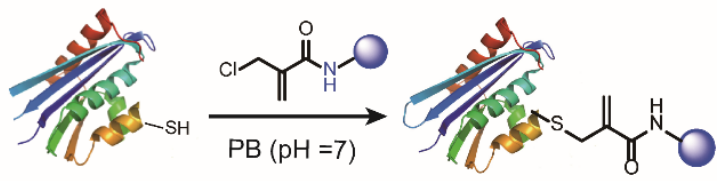

Ubiquitin

b

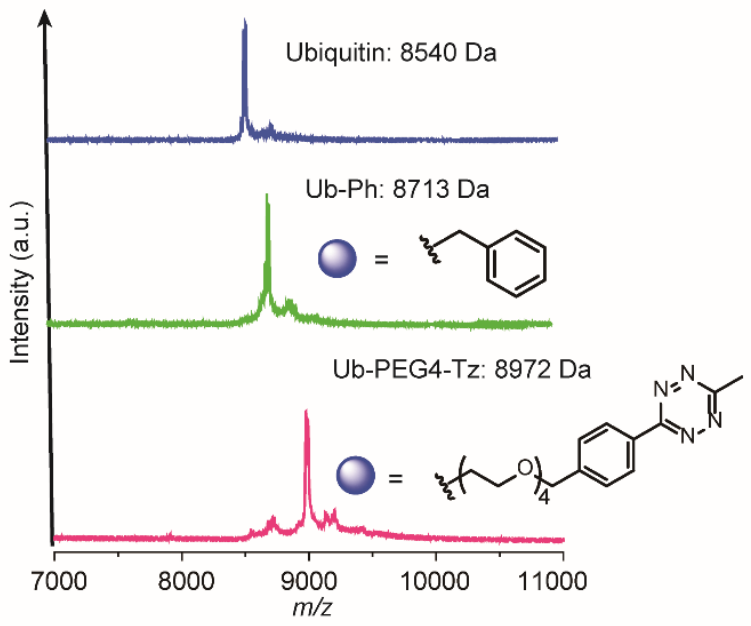

C

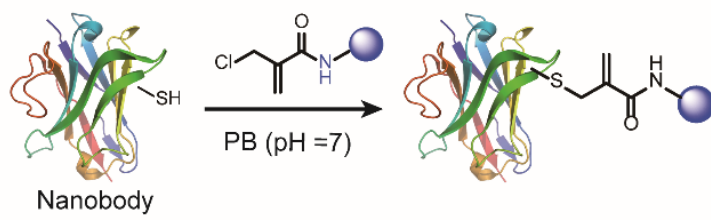

d

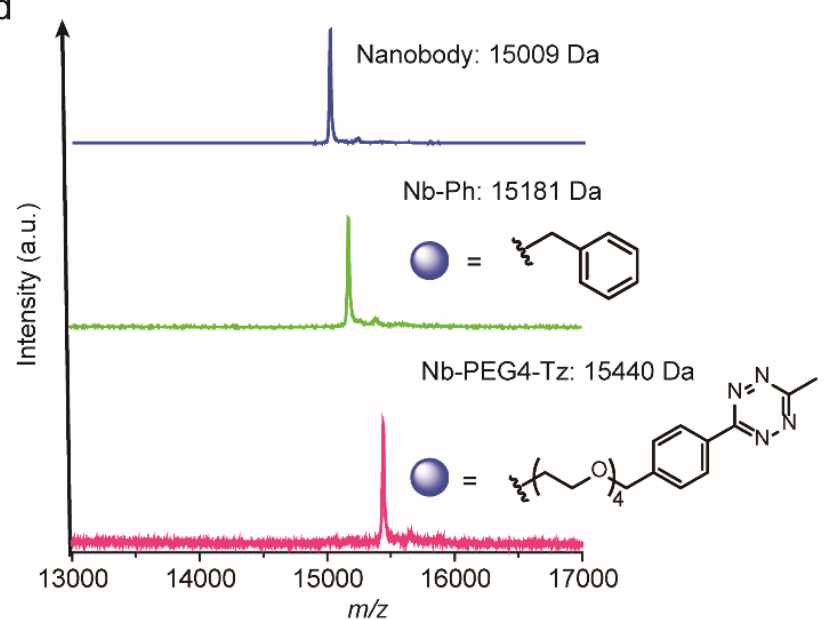

Fig. 5 (a) Site-selective modification of ubiquitin at cysteine site with two different functionalities: a phenyl and a tetrazine group. (b) Successful modification was proved by the MALDI-Tof-MS characterization with a peak at 8713 for Ub-Ph (calculated: $8714 \mathrm{Da}$, found: $8713 \mathrm{Da}$ ) and 8972 for Ub-PEG4-Tz (calculated: $8970 \mathrm{Da}$, found: $8972 \mathrm{Da}$ ). (c) Site-selective modification of anti-GFP nanobody at cysteine site with two different functionalities: a phenyl and a tetrazine group (d) MALDI-Tof-MS of the modified nanobody with a peak at 15181 for Nb-Ph (calculated: $15183 \mathrm{Da}$, found: $15181 \mathrm{Da}$ ) and 15440 for Nb-PEG4-Tz (calculated: $15438 \mathrm{Da}$, found: $15440 \mathrm{Da}$ )

\section{2-Chloromethyl acrylate reagents for disulfide modification}

Next, the feasibility of 2-chloromethyl acrylate for disulfide bond modification was evaluated on both peptide and protein substrates. The cyclic peptide hormone somatostatin (SST), which plays a key role in regulating the endocrine system and contains an accessible disulfide bond in its sequence ${ }^{42}$, was selected as model peptide (Fig. 6a). The disulfide bond in SST was first reduced by two equivalents of tris(2carboxyethyl)phosphine (TCEP) to generate the two free thiol groups in an ACN / PB mixture (1:10) at pH 7 , followed by incubation with 1.1 equivalents of compound $\mathbf{4}$ for overnight. HPLC of the crude reaction mixture between SST and compound 4 revealed good modification efficiency ( $91 \%$ based on HPLC quantification) (Fig. 6b). The isolated SST-Ph conjugate was also characterized by MALDI-Tof-MS showing successful functionalization (Fig. 6c). SST-Ph was further incubated with TCEP before the subsequent addition of the thiol reactive reagent, 4,4'-dithiodipyridine (4-DPS). HPLC analysis showed that SST-Ph remained intact without any observation of side reaction occurring with 4-DPS, thus indicating complete modification of the disulfide bond (Fig. 6b). In addition, the stability study revealed that the resultant SST-Ph conjugate showed 
reasonable stability up to 48 hours in acidic and basic conditions ( $\mathrm{pH}=6,7$ and 8$)$ (Fig. $6 \mathrm{~d})$ as well as in the presence of glutathione for 36 hours (Fig. S30). Since the 2-chloromethyl acrylate compounds do not contain aromatic groups in their scaffold, they have rather low $\log \mathrm{P}_{\mathrm{o} / \mathrm{w}}$ and thus provide better water-solubility than the reagents that contain phenyl groups, such as allyl sulfone reagents. This is particularly advantageous when modifying some therapeutic relevant proteins, which will suffer from aggregation issue if large amount of organic solvent is needed during the modification process. Therefore, the disulfide modification efficiency of SST with 2-chloromethyl acrylate and allyl sulfone reagents was evaluated and compared with using compound 8 and an allyl sulfone reagent (denoted as "IC-Tetrazine", which was developed by our group before $^{25}$ ) (Fig. 6a). Disulfide modification of SST with IC-Tetrazine required $40 \%$ ACN for solubilization, whereas less than $10 \%$ of $\mathrm{ACN}$ was needed to dissolve compound $\mathbf{8}$. More importantly, the modification efficiency of compound 8 was considerably higher ( $83 \%$ based on the quantification of HPLC peak for the reaction mixture) compared to IC-Tetrazine (67\%) (Fig. 6e). Besides SST, another therapeutic relevant cyclic peptide octreotide, an analogue of somatostatin with a longer biological half-life that is often applied in cancer diagnostics ${ }^{43}$, was also successfully functionalized with a coumarin motif under the similar reaction conditions mentioned above (Scheme S9). The MALDI-Tof-MS data confirmed the successful functionalization with a peak at 1364 corresponding to $[\mathrm{M}+\mathrm{H}]^{+}($Fig. S34).
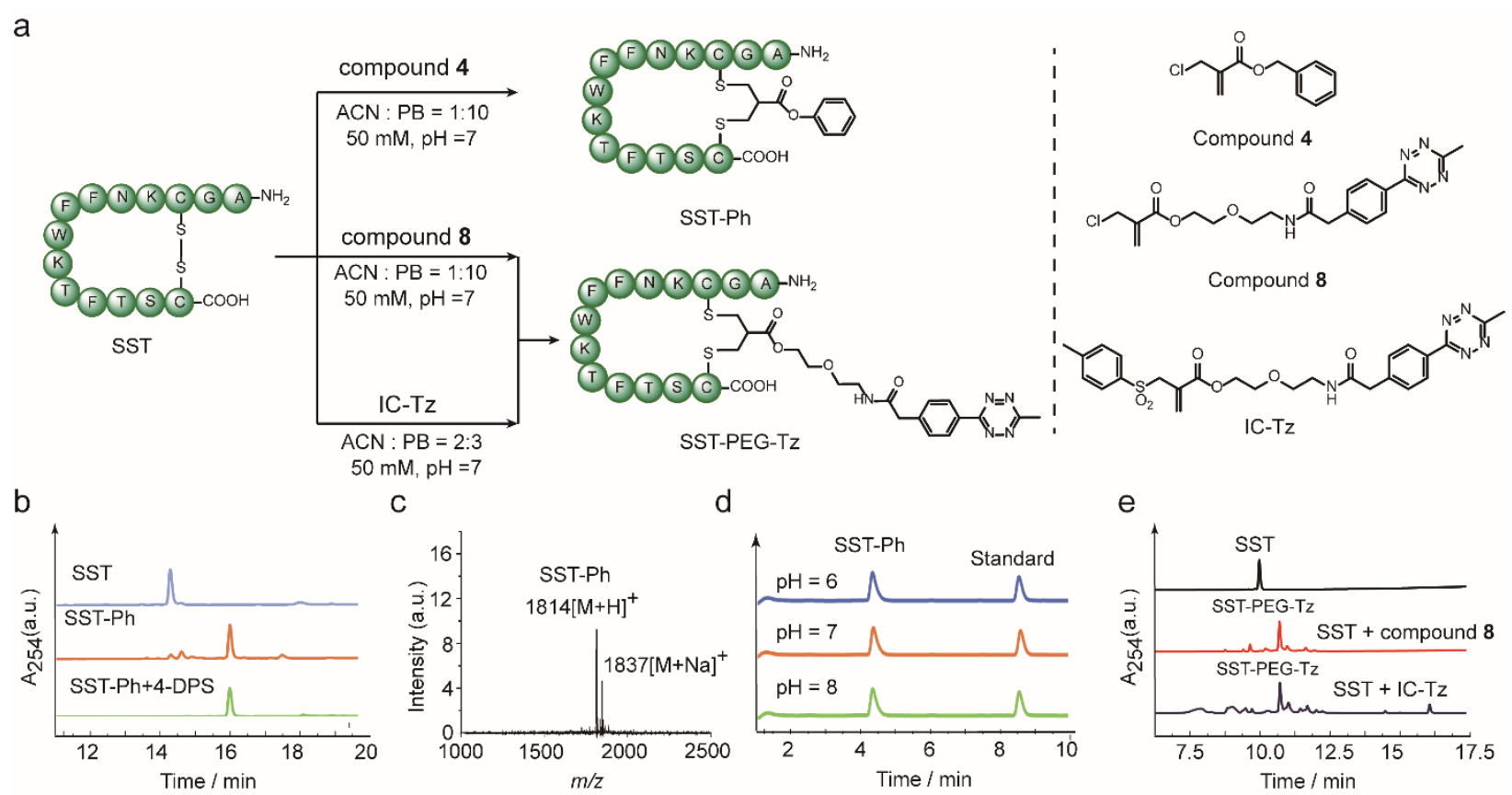

Fig. 6 (a) Modification of SST with compound 4, compound 8, and an allyl sulfone reagent (IC-Tz). (b) HPLC trace of the crude reaction mixture between SST and compound 4 as well as the mixture of SST-Ph and 4DPS; (c) MALDI-Tof-MS of the modified SST (SST-Ph) (calculated: $1814[\mathrm{M}+\mathrm{H}]^{+}$, found:1814 [M+H] $]^{+}, 1837$ 
$\left.[\mathrm{M}+\mathrm{Na}]^{+}\right)$; (d) Stability study of SST-Ph under three different $\mathrm{pH}$ conditions $(\mathrm{pH}=6,7$ and 8) for 36 hours; (e) HPLC trace of the crude reaction mixture between SST and compound 8 (or IC-Tz).

Subsequently, this new disulfide modification strategy was also evaluated on a much more complex protein substrate, the enzyme lysozyme (from hen egg white), in which the disulfide at C6 - C127 is predicted to be most solvent accessible among the four available disulfide bonds. ${ }^{23,44}$ To test the applicability of the 2chloromethyl acrylate compounds for disulfide modification, different functionalities were incorporated into lysozyme, such as a phenyl group, a fluorescent dye (coumarin), and a bioorthogonal tag (tetrazine group) (Fig. 7a). After using 2 equivalents of TCEP to break the most accessible disulfide bond, 1.1 equivalents of the 2-chloromethyl acrylate derivatives were added, and the reaction mixture were incubated at $50 \mathrm{mM} \mathrm{PB}$ $(\mathrm{pH} 7)$ overnight. Thereafter, the modified lysozyme derivatives (Ly-Ph, Ly-PEG-Cou and Ly-PEG-Tz) were purified by using $\mathrm{Hi}$ Trap hydrophobic interaction column with the isolated yields of $28 \%, 22 \%$ and $24 \%$, respectively. Notably, around $25-30 \%$ of native lysozyme was recovered after the purification, which can be used for further functionalization. MALDI-Tof-MS data of the three modified lysozymes derivatives confirmed their successful functionalization (Fig. 7b). In addition, the modified lysozymes did not show significant changes in their secondary structures compared to native lysozyme as shown in the circular dichroism spectra (Fig. S37).

Lysozyme is an antimicrobial enzyme that is capable of hydrolyzing the 1,4-beta-linkages in peptidoglycan of gram-positive bacterial cell walls, thus leading to the lysis of bacteria (Fig. 8a). Therefore, the catalytic activity of modified lysozyme was assessed by investigation of the absorbance change at 450 $\mathrm{nm}$ of Micrococcus lysodeikticus lyophilized cell suspensions over time, where the activity of the modified lysozyme is proportional to their capability to hydrolyze the bacterial cell walls. ${ }^{45}$ In comparison to native lysozyme, the disulfide-modified lysozyme Ly-PEG4-Tz retained $86 \%$ of its activity (Fig. 8b, calculation details shown in 8.3 in SI). In contrast, statistical modification of lysine residues of lysozyme using tetrazine $\mathrm{N}$ hydroxysuccinimide compounds (Scheme S11), which gave a heterogeneous mixture according to the MS data (Fig. S36), resulted in total loss of its catalytic activity (Fig. 8c, calculation details shown in 8.3 in SI). Hence, disulfide modification of proteins with 2-chloromethyl acrylate compounds represents an attractive approach to functionalize enzymatic proteins at distinct sites to preserve their catalytic activity. 


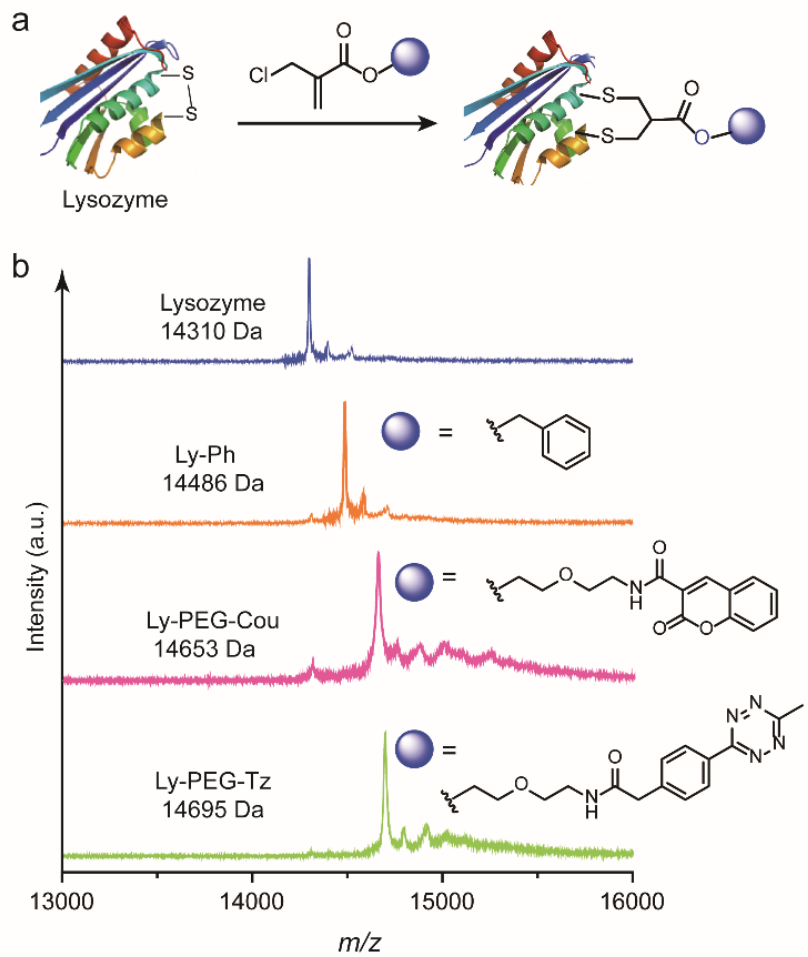

Fig. 7 (a) Site-selective disulfide modification of lysozyme with different functionalities. (b) MALDI-Tof-MS of modified lysozyme with different functionalities: Ly-Ph (calculated: $14485[\mathrm{M}+\mathrm{H}]^{+}$, found: $14486[\mathrm{M}+\mathrm{H}]^{+}$), LyPEG-Cou (calculated: $14654[\mathrm{M}+\mathrm{H}]^{+}$, found:14653 [M+H] $]^{+}$), Ly-PEG-Tz (calculated: $14694[\mathrm{M}+\mathrm{H}]^{+}$, found:14695 $[\mathrm{M}+\mathrm{H}]^{+}$)

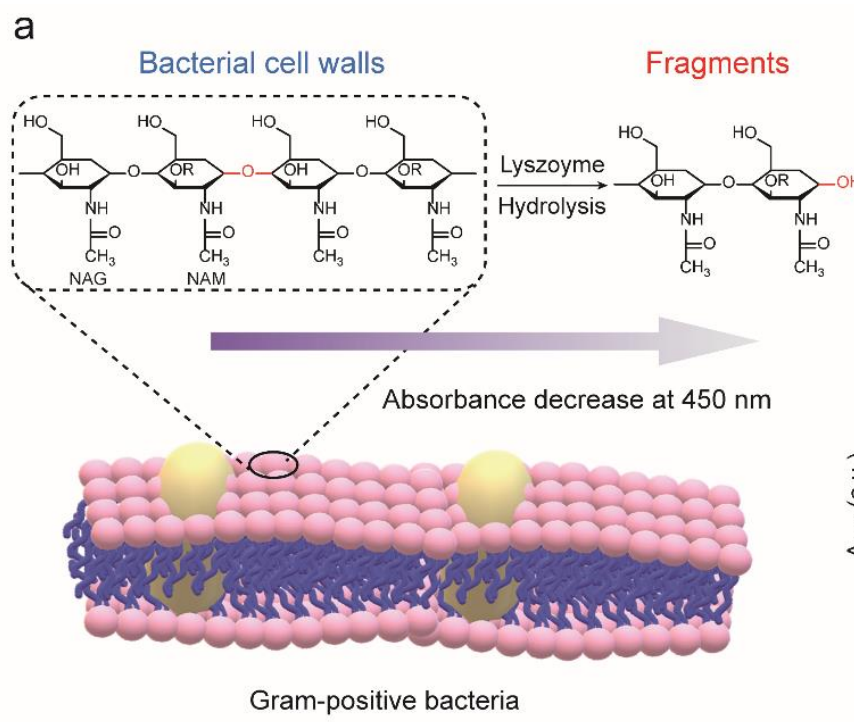

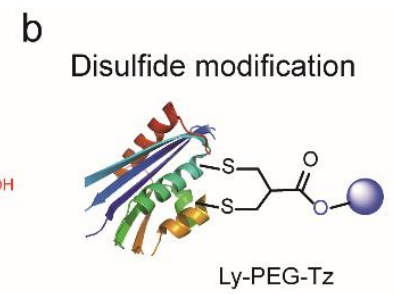

$86 \%$ preservation of the activity
C Statistical modification

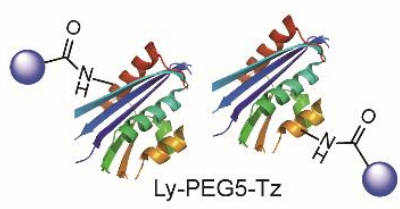

Total loss of the activity
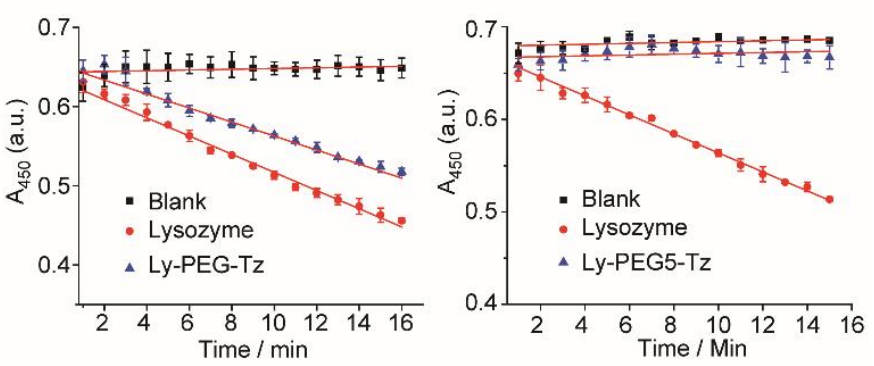

Fig. 8 (a) The hydrolysis of 1,4-beta-linkages of between $N$-acetyl-D-glucosamine (NAG) and $N$-acetylmuramic acid (NAM) residues in peptidoglycans of gram-positive bacterial cell walls. The absorbance of the cell suspension at $450 \mathrm{~nm}$ was monitored to evaluate their catalytic activity and the rate of absorbance decrease was proportional to its activity. (b) Compared to native lysozyme (red), disulfide-modified lysozyme retained $86 \%$ activity (blue). The calculation details are shown in Section 8.3 of the SI. (c) Statistical modified lysozyme based on NHS ester chemistry resulted in total loss of the catalytic activity. The calculation details were shown 
in SI

\section{Conclusion}

In conclusion, we report a simple yet versatile class of 2-chloromethyl acryl derivatives to achieve selective protein modification at cysteine or disulfide sites on demand. The synthesis of the reported 2chloromethyl acrylamide and acrylate compounds proceeds via a simple one-pot reaction based on the broadly accessible starting materials. Therefore, the synthesis is simple and highly practicable, so that it can be easily adapted in any lab even for the non-expert. The reactivity profile of the prepared bioconjugation reagents can be pre-determined in a user-defined fashion by selecting different end-group functionalities (either amino or hydroxyl groups) to obtain the respective 2-chloromethyl acrylate and acrylamide compounds.

Excellent labeling efficiency and high chemoselectivity of the 2-chloromethyl acrylamide compounds were demonstrated by the site-selective modification of cysteine residues in several model peptides as well as proteins. In contrast, 2-chloromethyl acrylate regents allow modification of disulfide-containing peptide and proteins, such as SST, octreotide, and lysozyme. In addition, our new approach offers the possibility for dual modification of proteins by capitalizing on the reactivity difference of the 2-chloromethyl acrylamide and acrylate compounds. In this way, one could envision protein dual functionalization at cysteine residues and disulfide bonds can be achieved in a stepwise fashion within one system. We believe that 2-chloromethyl acryl reagents presented herein greatly enrich the current available methodology toolbox for cysteine and disulfide modification, providing an easy access to the design and preparation of advanced protein conjugates for various biological, biophysical and medicinal applications.

\section{Acknowledgements}

The authors are grateful to the Max Planck Society, Deutsche Forschungsgemeinschaft (DFG, German Research Foundation) — Project number 316249678 - SFB 1279 (Projects A05, C01) for the funding. LX is grateful to the China Scholarship Council for a scholarship. We thank Dr. Zhixuan Zhou, Pia Winterwerber, Dr. Colette J Whitfield and Dr. David Yuen Wah Ng for the fruitful scientific discussion and the MPIP mass spectrometry group for the MALDI-TOF-MS measurements.

\section{References}

1. Krall, N., da Cruz, F. P., Boutureira, O. and Bernardes, G. J. L., Nat. Chem., 2016, 8, 103-113.

2. Akkapeddi, P., Azizi, S. A., Freedy, A. M., Cal, P., Gois, P. M. P. and Bernardes, G. J. L., Chem. Sci., 2016, 7, 2954-2963. 
3. Cal, P. M., Bernardes, G. J. and Gois, P. M., Angew. Chem. Int. Ed., 2014, 53, 10585-10587.

4. Spicer, C. D. and Davis, B. G., Nat. Commun., 2014, 5, 4740.

5. Stephanopoulos, N. and Francis, M. B., Nat. Chem. Biol., 2011, 7, 876-884.

6. Reddy, N. C., Kumar, M., Molla, R. and Rai, V., Org. Biomol. Chem., 2020, 18, 4669-4691.

7. Zhang, G., Zheng, S., Liu, H. and Chen, P. R., Chem. Soc. Rev., 2015, 44, 3405-3417.

8. Hoyt, E. A., Cal, P. M. S. D., Oliveira, B. L. and Bernardes, G. J. L., Nat. Rev. Chem., 2019, 3, 147-171.

9. Xu, L., Kuan, S. L. and Weil, T., Angew. Chem. Int. Ed., 2020, 60, 2-23.

10. Hou, Y. and Lu, H., Bioconjugate Chem., 2019, 30, 1604-1616.

11. Boutureira, O. and Bernardes, G. J. L., Chem. Rev., 2015, 115, 2174-2195.

12. Ravasco, J., Faustino, H., Trindade, A. and Gois, P. M. P., Chem. Eur. J., 2019, 25, 43-59.

13. Chalker, J. M., Bernardes, G. J., Lin, Y. A. and Davis, B. G., Chem. Asian. J., 2009, 4, 630-640.

14. Ochtrop, P. and Hackenberger, C. P. R., Curr. Opin. Chem. Biol., 2020, 58, 28-36.

15. Lu, J., Wang, H., Tian, Z., Hou, Y. and Lu, H., J. Am. Chem. Soc., 2020, 142, 1217-1221.

16. Kuan, S. L., Wang, T. and Weil, T., Chem. Eur. J., 2016, 22, 17112-17129.

17. Chudasama, V., Maruani, A. and Caddick, S., Nat. Chem., 2016, 8, 114-119.

18. Seki, H., Walsh, S. J., Bargh, J. D., Parker, J. S., Carroll, J. and Spring, D. R., Chem. Sci., 2021.

19. Bernardim, B., Cal, P. M., Matos, M. J., Oliveira, B. L., Martinez-Saez, N., Albuquerque, I. S., Perkins, E., Corzana, F., Burtoloso, A. C., Jimenez-Oses, G. and Bernardes, G. J., Nat. Commun., 2016, 7, 13128.

20. Baumann, A. L., Schwagerus, S., Broi, K., Kemnitz-Hassanin, K., Stieger, C. E., Trieloff, N., Schmieder, P. and Hackenberger, C. P. R., J. Am. Chem. Soc., 2020, 142, 9544-9552.

21. Zhang, C., Dai, P., Vinogradov, A. A., Gates, Z. P. and Pentelute, B. L., Angew. Chem. Int. Ed., 2018, 57, 64596463.

22. Choi, H., Kim, M., Jang, J. and Hong, S., Angew. Chem. Int. Ed., 2020, 59, 22514-22522.

23. Wang, T., Riegger, A., Lamla, M., Wiese, S., Oeckl, P., Otto, M., Wu, Y., Fischer, S., Barth, H., Kuan, S. L. and Weil, T., Chem. Sci., 2016, 7, 3234-3239.

24. Shaunak, S., Godwin, A., Choi, J.-W., Balan, S., Pedone, E., Vijayarangam, D., Heidelberger, S., Teo, I., Zloh, M. and Brocchini, S., Nat. Chem. Biol., 2006, 2, 312-313.

25. Xu, L., Raabe, M., Zegota, M. M., Nogueira, J. C. F., Chudasama, V., Kuan, S. L. and Weil, T., Org. Biomol. Chem., 2020, 18, 1140-1147.

26. Smith, M. E. B., Schumacher, F. F., Ryan, C. P., Tedaldi, L. M., Papaioannou, D., Waksman, G., Caddick, S. and Baker, J. R., J. Am. Chem. Soc., 2010, 132, 1960-1965.

27. Maruani, A., Smith, M. E., Miranda, E., Chester, K. A., Chudasama, V. and Caddick, S., Nat. Commun., 2015, 6. 6645 .

28. Walsh, S. J., Omarjee, S., Galloway, W., Kwan, T. T., Sore, H. F., Parker, J. S., Hyvonen, M., Carroll, J. S. and Spring, D. R., Chem. Sci., 2019, 10, 694-700.

29. Martinez-Saez, N., Sun, S., Oldrini, D., Sormanni, P., Boutureira, O., Carboni, F., Companon, I., Deery, M. J., Vendruscolo, M., Corzana, F., Adamo, R. and Bernardes, G. J. L., Angew. Chem. Int. Ed., 2017, 56, 14963-14967. 30. Zhang, Y., Zang, C., An, G., Shang, M., Cui, Z., Chen, G., Xi, Z. and Zhou, C., Nat. Commun., 2020, 11, 1015. 31. Stieger, C. E., Franz, L., Korlin, F. and Hackenberger, C. P. R., Angew. Chem. Int. Ed., 2021, 60, 1-7.

32. Liu, Y., Lai, Z., Yang, P., Xu, Y., Zhang, W., Liu, B., Lu, M., Chang, H., Ding, T. and Xu, H., RSC Advances. 2017, 7, 43104-43113.

33. Alexander Bohme, D. T., Albrecht Paschke, and Gerrit Schrmann, Chem. Res. Toxicol., 2009, 22, 742-750.

34. Saito, F., Noda, H. and Bode, J. W., ACS Chem Biol. 2015, 10, 1026-1033.

35. Kasper, M. A., Stengl, A., Ochtrop, P., Gerlach, M., Stoschek, T., Schumacher, D., Helma, J., Penkert, M., 
Krause, E., Leonhardt, H. and Hackenberger, C. P. R., Angew. Chem. Int. Ed., 2019, 58, 11631-11636.

36. Ulrich, S., Boturyn, D., Marra, A., Renaudet, O. and Dumy, P., Chem. Eur. J., 2014, 20, 34-41.

37. Dirksen, A., Hackeng, T. M. and Dawson, P. E., Angew. Chem. Int. Ed., 2006, 45, 7581-7584.

38. John C. Jewett, E. M. S., and Carolyn R. Bertozzi, J. Am. Chem. Soc., 2010, 132, 3688-3690.

39. Zirafi, O., Kim, K. A., Standker, L., Mohr, K. B., Sauter, D., Heigele, A., Kluge, S. F., Wiercinska, E., Chudziak, D., Richter, R., Moepps, B., Gierschik, P., Vas, V., Geiger, H., Lamla, M., Weil, T., Burster, T., Zgraja, A., Daubeuf, F., Frossard, N., Hachet-Haas, M., Heunisch, F., Reichetzeder, C., Galzi, J. L., Perez-Castells, J., CanalesMayordomo, A., Jimenez-Barbero, J., Gimenez-Gallego, G., Schneider, M., Shorter, J., Telenti, A., Hocher, B., Forssmann, W. G., Bonig, H., Kirchhoff, F. and Munch, J., Cell Rep. 2015, 11, 737-747.

40. Hansen, R. E., Østergaard, H., Nørgaard, P. and Winther, J. R., Anal. Biochem., 2007, 363, 77-82.

41. Fontaine, S. D., Reid, R., Robinson, L., Ashley, G. W. and Santi, D. V., Bioconjugate Chem., 2015, 26, 145-152.

42. Kuan, S. L., Fischer, S., Hafner, S., Wang, T., Syrovets, T., Liu, W., Tokura, Y., Ng, D. Y. W., Riegger, A., Förtsch, C., Jäger, D., Barth, T. F. E., Simmet, T., Barth, H. and Weil, T., Adv. Sci., 2018, 1701036.

43. Lamberts, S. W. J. and Hofland, L. J., Eur J Endocrinol. 2019, 181, R173-R183.

44. Zegota, M., Wang, T., Seidler, C., Ng, D. Y. W., Kuan, S. L. and Weil, T., Bioconjugate Chem., 2018, 29, 26652670.

45. Shugar, D., Biochim. Biophys. Acta. 1952, 8, 302-309. 